\title{
NEUROCIÊNCIA NA EDUCAÇÃO ESPECIAL: DOS NEUROMITOS ÀS PRÁTICAS PEDAGÓGICAS BASEADAS EM EVIDÊNCIAS
}

\author{
NEUROCIENCIA EN LA EDUCACIÓN ESPECIAL: DE NEUROMITOS A \\ PRÁCTICAS PEDAGÓGICAS BASADAS EN LA EVIDENCIA
NEUROSCIENCE IN SPECIAL EDUCATION: FROM NEUROMYTHS TO EVIDENCE-BASED PEDAGOGICAL PRACTICES

\author{
Daniel Traina GAMA ${ }^{1}$ \\ Marcela de Castro FERRACIOLI ${ }^{2}$
}

RESUMO: O presente documento tem por objetivo apresentar uma discussão sobre as fundamentações lógica e teórica que subsidiam a disciplina de neurociência do curso de Especialização em Educação Especial da Faculdade de Educação/UFGD. A neurociência tem avançado de forma importante nos conhecimentos relacionados à educação e educação especial. No entanto, profissionais da área da educação têm dificuldades na interpretação e utilização desses conhecimentos nas práticas pedagógicas. Verifica-se que professores apresentam prevalência na crença de neuromitos, prejudicando suas práticas educacionais. Portanto, objetiva-se com a disciplina de neurociência a desconstrução de neuromitos e aprendizado das estruturas e funções neuroanatômicas e seus processos subjacentes nos diferentes níveis de análise da neurociência pelos alunos. Relacionando e discutindo os princípios aprendidos com a Educação Especial e o Atendimento Educacional Especializado.

PALAVRAS-CHAVE: Neurociência. Educação. Educação especial. Atendimento Educacional Especializado.

RESUMEN: Este artículo tiene como objetivo presentar una discusión sobre los fundamentos lógicos y teóricos que apoyan la disciplina de neurociencia del Curso de Especialización en Educación Especial de la Facultad de Educación / UFGD. La neurociencia ha avanzado significativamente en el conocimiento relacionado con la educación y la educación especial. Sin embargo, los profesionales de la educación tienen dificultades para interpretar y utilizar este conocimiento en las prácticas pedagógicas. Se verifica que los maestros tienen prevalencia en la creencia de neuromitos, perjudicando sus prácticas educativas. Por lo tanto, el objetivo de la disciplina de neurociencia es deconstruir neuromitas y aprender las estructuras y funciones neuroanatómicas y sus procesos subyacentes en los diferentes niveles de análisis de neurociencia por parte de los estudiantes. Relacionar y discutir los principios aprendidos con Educación Especial y Atención Educativa Especializada.

PALABRAS CLAVE: Neurociencia. Educación. Educación especial. Servicio Educativo Especializado.

1 Universidade Federal da Grande Dourados (UFGD), Dourados - MS - Brasil. Docente. Doutor em Desenvolvimento Humano e Tecnologias (UNESP/Rio Claro). ORCID: http://orcid.org/0000-0003-3984-326X. E-mail: gamadaniel@ hotmail.com

${ }^{2}$ Universidade Federal do Ceará (UFC), Fortaleza - CE - Brasil. Docente. Doutora em Desenvolvimento Humano e Tecnologias (UNESP/Rio Claro). ORCID: https://orcid.org/0000-0003-1782-691X. E-mail: marcelaferracioli@gmail.com 
ABSTRACT: The aim of this paper is to present a discussion about the logical and theoretical foundations that support the neuroscience discipline of the Special Education Specialization course of the Education College/UFGD. Neuroscience has advanced significantly in the knowledge related to education and special education. However professionals in the field of education present difficulties in the interpretation and use of this knowledge in pedagogical practices. It is verified that teachers present prevalence in the belief of neuromyths, damaging their educational practices. Therefore, the goals of the neuroscience discipline are the deconstruction of neuromyths and learning of neuroanatomic structures and functions and their underlying processes at different levels of neuroscience analysis by students. Linking and discussing the principles learned with Special Education and Specialized Educational Attendance.

KEYWORDS: Neuroscience. Education. Special Education. Specialized Educational Attendance.

\section{Introdução}

O curso de Especialização em Educação Especial (EEE), vinculado à Faculdade de Educação (FAED) da Universidade Federal da Grande Dourados (UFGD), tem como principal objetivo formar profissionais para o Atendimento Educacional Especializado para atuação em escolas comuns e centros especializados. Dentre as principais capacitações desenvolvidas no curso de EEE destacam-se: desenvolvimento de habilidades específicas de comunicação, língua e linguagem, mobilidade e locomoção, práticas pedagógicas para as ações da Educação Especial; assessorias aos professores e alunos na classe comum da escola, além de estudos e desenvolvimento de recursos técnicos e tecnológicos, envolvendo espaços escolares e não escolares em uma perspectiva inclusiva (UNIVERSIDADE FEDERAL DA GRANDE DOURADOS, 2019).

O programa do curso foi desenvolvido com base no atendimento ao Decreto n. $^{\circ}$ 7.611, da Presidência da República, de 17 de novembro de 2011, que dispõe sobre a Educação Especial e o Atendimento Educacional Especializado (AEE). Nesse documento, o AEE é caracterizado pelo conjunto de atividades, recursos de acessibilidade e pedagógicos organizados institucional e continuamente para o público-alvo da Educação Especial, alunos com deficiências, com transtornos globais do desenvolvimento e com altas habilidades e superdotação.

Mais especificamente, no mesmo decreto, e de interesse para o presente relato, o artigo $3 .^{\circ}$ lista os objetivos do AEE:

I - prover condições de acesso, participação e aprendizagem no ensino regular e garantir serviços de apoio especializados de acordo com as necessidades individuais dos estudantes; 
II - garantir a transversalidade das ações da educação especial no ensino regular;

III - fomentar o desenvolvimento de recursos didáticos e pedagógicos que eliminem as barreiras no processo de ensino e aprendizagem; e

IV - assegurar condições para a continuidade de estudos nos demais níveis, etapas e modalidades de ensino.

Portanto, considerando o contexto social ainda carente de especialistas em AEE, o processo formativo do curso de EEE inclui o estudo das bases epistemológicas do desenvolvimento humano nos seus diferentes domínios e de práticas pedagógicas voltadas para alunos especiais em uma perspectiva inclusiva. A formação profissional acontece sob a luz da perspectiva pós-crítica de educação e de teoria de currículo, por meio de práticas educativas ativas e inclusivas, e o conhecimento é construído e reconstruído pelo fazer, sentir e pensar com atitudes relacionais dos conteúdos abordados no programa com o fenômeno estudado e a atuação profissional. Assim, essa formação privilegia a interdependência, a complementaridade, a conectividade dos conteúdos curriculares e experiências dos alunos (UNIVERSIDADE FEDERAL DA GRANDE DOURADOS, 2019).

Relacionada às bases epistemológicas indispensáveis para atingir os objetivos da educação como um todo e, consequentemente, da Educação Especial e do AEE, a neurociência tem como principal objetivo o entendimento do sistema nervoso por meio do estudo dos processos que possibilitam suas funções, tais como uma pessoa percebe e age no ambiente, aprende e memoriza novas informações, cria, se comunica e se expressa (BEAR; CONNORS; PARADISO, 2007). Nesse sentido, o objetivo do presente artigo é apresentar uma discussão sobre as fundamentações lógica e teórica que subsidiam a disciplina de neurociência do curso EEE da FAED/UFGD. Portanto, o presente artigo foi organizado em três tópicos de discussão: neuromitos e educação, princípios de neurociência na educação e neurociência na educação especial.

\section{Neuromitos e educação}

A neurociência tem recebido grande atenção da sociedade em razão das descobertas reveladoras sobre processos envolvidos nas funções do sistema nervoso, na maioria das vezes, apresentadas em periódicos de alto fator de impacto científico (RACINE et al., 2010). Diante disso, a mídia popular e desenvolvedores de produtos relacionados à neurociência e educação costumam reportar muitos desses estudos de forma superficial, beneficiando-se das suas principais conclusões de maneira simplória, falhando na qualidade da informação, providenciando informações fragmentadas e descontextualizadas dos resultados dos estudos. Esse formato de informação leva o público a fazer interpretações e generalizações 
equivocadas, colaborando na criação de neuromitos (DEKKER et al., 2012; GOSWAMI, 2006). Os neuromitos são crenças equivocadas, com consequentes tentativas precipitadas de aplicação deliberada de práticas fundamentadas em fragmentos de conclusões de estudos em neurociência de maneira generalizada e fora de contexto (HOWARD-JONES, 2014).

No tocante à educação, o despertar das possibilidades da aplicação dos conhecimentos em neurociência para o desenvolvimento de práticas pedagógicas por professores é um fenômeno que começou a crescer na década de 1990, principalmente com o grande número de estudos fundamentados em neuroimagem, os quais permitiram uma nova perspectiva na análise dos processos neurais por meio da visualização das áreas cerebrais ativadas, enquanto funções executivas são realizadas (DEKKER et al., 2012). No entanto, em virtude de os estudos em neurociência serem realizados em ambientes de laboratório altamente controlados e investigarem situações-problema específicas, a transferência desses conhecimentos torna-se difícil e de contestada aplicação nas práticas escolares (ANSARI; COCH; DE SMEDT, 2011).

Essa grande lacuna existente entre a neurociência e as práticas pedagógicas, por sua vez, também permite aos professores adotar equivocadas estratégias de práticas pedagógicas supostamente fundamentadas em evidências dessa área (GOSWAMI, 2006). Na realidade, há uma avalanche comercial de pacotes e programas educacionais que se dizem, erroneamente, bem fundamentados em neurociência. De acordo com Goswami (2006), essas desinformações necessitam ser eliminadas da educação, pois ignoram informações relevantes da neurociência que realmente poderiam ser utilizadas na educação, além de fazerem professores e instituições perderem energia, tempo e investimento com estratégias e ferramentas sem a real comprovação de serem eficazes para atingir seus objetivos (PAPADATOU-PASTOU; HALIOU; VLACHOS, 2017).

Em geral, uma das causas para que os resultados de estudos em neurociência sejam mal interpretados e, consequentemente, utilizados equivocadamente nas práticas pedagógicas é o fato de a maioria dos professores não dominar conhecimentos básicos nessa área (HERCULANO-HOUZEL, 2002). Corroborando esse fato, Gleichgerrcht et al. (2015) mostraram, em uma pesquisa com 3.451 professores da América Latina, de todos os níveis educacionais, alta prevalência de professores com conhecimentos limitados em neurociências e crença em neuromitos.

Mais interessante ainda é o fato de professores do Reino Unido e da Holanda, apesar de apresentarem um bom conhecimento em neurociência, acreditarem em neuromitos populares para a adoção de práticas pedagógicas (DEKKER et al., 2012). Entre os neuromitos destacam-se as crenças de que: as pessoas aprendem melhor quando recebem informação de 
modo preferido (pelo modo sensorial de preferência, tal como o visual, auditivo ou cinestésico); diferenças de dominância hemisférica (direita ou esquerda) ajudam a explicar diferenças entre aprendizes; exercícios de coordenação intermembros ajudam a melhorar a integração da função hemisférica direita e esquerda; um ambiente rico em estímulos ajuda no melhor desenvolvimento cerebral; as pessoas utilizam apenas $10 \%$ de suas capacidades cerebrais (HOWARD-JONES, 2014).

Provavelmente, o conhecimento limitado de professores em neurociências acontece porque a maioria deles não teve, ou teve apenas superficialmente, conteúdos curriculares vinculados a essa área em seus cursos de formação no ensino superior. Grossi, Lopes e Couto (2014) analisaram a presença de disciplinas de neurociência e correlatas nos cursos de Pedagogia no Brasil e verificaram que somente $6,25 \%$ dos currículos dos cursos apresentaram essas disciplinas. Os autores consultaram 352 matrizes curriculares de cursos credenciados no Ministério da Educação e Cultura, de instituições de ensino públicas e privadas de todas as regiões do País.

Levando em consideração os importantes avanços em neurociência relacionados à educação, o potencial de contribuição desses conhecimentos para prática pedagógica e a quantidade de desinformação disponível relacionada aos temas, o primeiro passo para resolução dos problemas apontados é a capacitação dos professores para o entendimento crítico e construtivo para educação dos princípios em neurociência. Para tanto, é necessário que os professores em formação e formados aprendam sobre as estruturas e funções neuroanatômicas e seus processos subjacentes nos diferentes níveis de análise da neurociência para o desenvolvimento humano, em especial os concernentes à aprendizagem e educação em populações típicas e especiais.

\section{Princípios de neurociência na educação}

Em contrapartida aos neuromitos na educação, princípios em neurociência bem consolidados empiricamente foram estabelecidos para beneficiar a prática educacional. A seguir, serão apresentadas uma revisão e discussão das principais descobertas e princípios em neurociência para a educação, sugeridas pela publicação da Real Sociedade de Londres (2011):

\section{- O cérebro possui alta capacidade adaptativa ou de plasticidade}

O cérebro tem grande potencial de adaptabilidade e está em constante processo de mudança, um processo denominado plasticidade cerebral ou neuroplasticidade (DRAGANSKI et al., 2004). Esse efeito é conhecido como dependente de experiências, ou 
seja, em que as conexões de neurônios são fortalecidas ou enfraquecidas de acordo com experiências de aprendizagem de habilidades específicas (LINDENBERGER; WENGER; LÖVDÉN, 2017). As relações neuronais são armazenadas na memória, tornando-se mais estáveis e eficientes à medida que a aprendizagem acontece. Dessa forma, o cérebro pode se preparar para eventos futuros similares. A plasticidade cerebral apresenta períodos mais sensíveis na infância e adolescência e diminui sua capacidade no avançar da idade, porém a capacidade de adaptabilidade cerebral para a aprendizagem se mantém durante todo o ciclo vital (DE LANGE et al., 2018), e a dinâmica dessas mudanças apresenta diferenças de acordo com a função cognitiva e com as áreas cerebrais. No entanto, existem limites para o potencial de plasticidade, bem como diferenças individuais e contextuais. Evidências empíricas sugerem limites relacionados à predisposição interna e à estimulação ambiental que podem afetar a aprendizagem (THOMAS, 2003).

\section{- Tanto a genética quanto as influências ambientais afetam a aprendizagem cerebral}

As pessoas apresentam diferentes respostas ao processo de aprendizagem em razão de suas diferenças individuais, e os genes e o ambiente desempenham papel fundamental nessas diferenças. Por exemplo, estudos realizados com gêmeos monozigóticos mostraram mais similaridades em capacidades de leitura e matemáticas entre eles do que em gêmeos não idênticos (OLSON, 2018). Apesar disso, a neurociência genética é capaz de explicar apenas algumas predisposições favoráveis ou desfavoráveis para algumas funções, enquanto os resultados da aprendizagem são altamente influenciados por meio das experiências ou qualidade na educação (VON STUMM; PLOMIN, 2018).

\section{- A resposta cerebral para recompensa é influenciada por grau de expectativa e incerteza}

Pesquisas em neurociência mostraram que a resposta cerebral para recompensa é influenciada por diferentes fatores, por exemplo: a redução de erros de predição nos resultados durante o processo de aprendizagem por si só garante recompensa satisfatória; e o grau de incerteza e curiosidade sobre a recompensa recebida influencia a magnitude da resposta neural gerada, que, por sua vez, define a importância da recompensa (MARVIN; SHOHAMY, 2016). O entendimento dos mecanismos de recompensas e motivação pode contribuir para o desenvolvimento de estratégias pedagógicas efetivas para a educação (HIDI, 2016). 


\section{- O cérebro possui mecanismos autorregulatórios}

Pesquisas em neurociência têm demonstrado os mecanismos cerebrais para a autorregulação e autocontrole na inibição de comportamentos impulsivos ou de recompensa imediata. Tem-se observado que a capacidade inibitória de recompensa imediata é desenvolvida desde a infância até o começo da vida adulta, provavelmente devido ao desenvolvimento do córtex pré-frontal, área cerebral associada, principalmente, à aprendizagem, criatividade, raciocínio lógico e tomada de decisão, que continua em pleno processo de mudanças em termos de estrutura e função até o final da adolescência (LOPEZ et al., 2017; RAVER; BLAIR, 2016). No entanto, verificam-se muitas diferenças individuais na capacidade de inibição de comportamentos de recompensa imediata, e melhores resultados inibitórios na infância estão relacionados com melhores resultados educacionais na adolescência. Esses resultados são importantes, pois esses comportamentos inibitórios, por sua vez, também podem ser aprendidos em benefício da educação (DUMONTHEIL, 2016).

- Existem muitas evidências de fatores que contribuem de forma significativa para o desenvolvimento cognitivo

A educação, por exemplo, é um fator que contribui de forma significativa para o desenvolvimento cerebral e manutenção de suas funções. Pesquisas demonstraram correlação negativa entre o engajamento educacional e a apresentação de demência e declínio cognitivo no envelhecimento (WILSON et al., 2009; ZAHODNE; STERN; MANLY, 2015). A prática de atividades físicas regulares (DUZEL; VAN PRAAG; SENDTNER, 2016), a qualidade e privação do sono (KRAUSE et al., 2017) e a nutrição (GEORGIEFF; BRUNETTE; TRAN, 2015) são exemplos de hábitos voltados para a saúde que determinam o bom desenvolvimento cognitivo. Em contraposição, o uso deliberado de fármacos nootrópicos, considerados potencializadores cognitivos, para pessoas com função cerebral típica, pode comprometer a função cognitiva, uma vez que seus efeitos a longo prazo ainda não estão bem explicado (BRÜHL; SAHAKIAN, 2016).

Tendo em vista a quantidade e a qualidade de informações que relacionam a neurociência com a educação, profissionais da educação podem se beneficiar desses conhecimentos para o desenvolvimento de políticas públicas e estratégias no atendimento de suas populações. No entanto, por essas informações serem ainda consideradas recentes e em desenvolvimento científico para criação de novos paradigmas, os profissionais da educação necessitam dominar os conhecimentos fundamentais em neurociência para realização de análises críticas e reflexivas desse vasto e rico conteúdo informacional. 


\section{Neurociência na educação especial}

A educação especial também vem sendo foco de estudos em neurociência. Esses conhecimentos podem ser muito bem utilizados para que profissionais da educação tenham melhores entendimentos sobre as causas das dificuldades apresentadas por populações especiais, bem como sobre os processos não típicos de aprendizagem dessas populações (PAPADATOU-PASTOU; HALIOU; VLACHOS, 2017). Acredita-se que, por meio do melhor entendimento desses processos, professores podem elaborar estratégias para amenizar ou até mesmo superar as dificuldades de aprendizagem dessas populações.

Entretanto, é importante que os profissionais realizem algumas reflexões sobre os princípios em neurociências para educação. Por exemplo, uma dificuldade apresentada em práticas pedagógicas para populações especiais é assumir estereótipos comportamentais em torno de uma população específica, muitas vezes com base em poucas experiências do profissional com determinada população. O princípio da grande presença de diferenças individuais e contextuais na adaptação/plasticidade cerebral, ou seja, no processo de aprendizagem (THOMAS, 2003), também deve ser respeitado para uma determinada população especial. Assim, mesmo que indivíduos apresentem uma mesma condição, eles provavelmente terão comportamentos diferentes e demandarão atenção individual no que se refere às suas particularidades.

Sabe-se que, o quanto antes uma condição especial é descoberta, melhores são as oportunidades de as crianças receberem atendimento especializado a fim de amenizar ou superar suas dificuldades (CIONI; INGUAGGIATO; SGANDURRA, 2016). Com a realização de pesquisas voltadas para populações especiais, foram desenvolvidas técnicas de avaliação que, por meio de testes clínicos específicos, são capazes de identificar condições especiais, tais como testes de desenvolvimento motor e psicológicos, exames neurológicos, exames de neuroimagem e exames genéticos.

Cioni et al. (2016) destacam que, para os programas de intervenção de populações especiais na infância serem maximamente bem-sucedidos, são necessárias algumas condições, por exemplo, a intervenção deve começar cedo, ser intensa, ativa, pensada para cada indivíduo e centrada na família. No entanto, os autores relatam que intervenções que atendem todas essas condições são caras, de difícil acesso pela maioria da população. Portanto, estratégias de intervenção fundamentadas em tecnologias com assistência remota à família pode ser uma alternativa eficaz no AEE.

Levando em consideração que o conhecimento educacional é desenvolvido de forma interdependente e complementar, entende-se que fraquezas ou dificuldades em determinadas 
capacidades podem ser aspectos limitadores de taxa no processo de aquisição de novas habilidades pelas crianças. Na tentativa de entendimento de processos fortes e fracos de desenvolvimento, pesquisadores apresentaram uma ferramenta de avaliação de crianças com deficiências denominada processing strenght and weakness - PSW (HALE et al., 2010). Hale et al. (2016) ressaltam que a utilização da PSW foi eficiente para identificar o nível de capacidade em diferentes aspectos, subsidiando diagnósticos e atendimento especializado para crianças nas áreas de leitura, matemática, expressão escrita e comportamento emotivo.

A utilização de ferramentas como a PSW na educação pode colaborar para desmitificar estereótipos criados em torno de determinadas condições, permitindo o entendimento individual das capacidades das crianças e, por sua vez, um atendimento especializado fundamentado em torno de suas capacidades funcionais identificadas. As evidências em neurociência demonstram que crianças com condições especiais têm deficiências, e não atrasos no desenvolvimento. Portanto, estratégias para remediar as deficiências parecem ser melhores alternativas de intervenção para elas, no sentido da construção de suas habilidades na educação, em vez de intervenção baseada na compensação desses déficits (HALE et al., 2016).

Para Hale et al. (2016), remediar as deficiências está relacionado primeiro com a identificação dos déficits limitadores de desenvolvimento em crianças especiais, tais como de atenção, de linguagem, de processamento de informação, de memória de trabalho, de habilidades psicomotoras, para que esses déficits sejam atenuados ou eliminados por estratégias de intervenção. Prospecta-se que esses déficits, uma vez remediados, não sejam mais limitadores de construção de novas habilidades em crianças com deficiência. Essa abordagem de intervenção se contrapõe às estratégias de compensação das dificuldades, em que alternativas de práticas são criadas adaptadas aos déficits apresentados, ou seja, sem a necessidade de atenuação ou superação desses déficits. Segundo os referidos autores, essas abordagens compensatórias não são eficientes, pois não resolvem problemas futuros de aprendizagem.

Considerando o conteúdo apresentado, verificam-se importantes aspectos a serem considerados no tocante ao impacto da neurociência na educação especial. Primeiramente, a desinformação provocada pela divulgação superficial, fragmentada e descontextualizada dos conhecimentos em neurociência é perigosa para a educação como um todo, afetando também o AEE. Em contrapartida, o grande volume e a boa qualidade de informações científicas relacionando as áreas de neurociência e educação representam ótimas oportunidades para os profissionais de educação subsidiarem suas práticas em evidências científicas. A perspectiva futura para a Educação Especial é que novos paradigmas educacionais sejam criados no sentido de beneficiar o AEE. 


\section{Considerações finais}

A pesquisa em neurociência na educação especial tem avançado de forma importante e, para otimizar o AEE, os profissionais da área devem estar capacitados e atualizados com relação às possibilidades apresentadas nos meios de divulgação da produção desses conhecimentos. Portanto, a disciplina de neurociência do curso de Especialização em Educação Especial da FAED/UFGD apresenta como objetivos principais: (i) desconstrução de neuromitos populares na área de educação; (ii) construção do conhecimento em neurociência por meio do estudo crítico, analítico e relacional das estruturas e funções neuroanatômicas e seus processos subjacentes nos diferentes níveis de análise da neurociência; (iii) estudo e discussão dos princípios em neurociência relacionados à educação; e (iv) estudo e discussão dos princípios em neurociência aplicados à educação especial e ao atendimento educacional especializado. Aspira-se que o profissional formado no curso de EEE esteja capacitado para identificar neuromitos na educação e entenda a neurociência suficientemente, a ponto de subsidiar sua prática baseada em evidências científicas de boa qualidade.

\section{REFERÊNCIAS}

ANSARI, D.; COCH, D.; DE SMEDT, B. Connecting education and cognitive neuroscience: where will the journey take us? Educational Philosophy and Theory, v. 43, n. 1, p. 37-42, 2011.

BEAR, M. F.; CONNORS, B. W.; PARADISO, M. A. Neuroscience: exploring the brain. 3rd. ed. Philadelphia: Lippincott Williams \& Wilkins, 2007.

BRASIL. Decreto n. ${ }^{0}$ 7.611, de 17 de novembro de 2011. Presidência da República, Brasília, 2011.

BRÜHL, A. B.; SAHAKIAN, B. J. Drugs, games, and devices for enhancing cognition: implications for work and society. Annals of the New York Academy of Sciences, v. 1369, n. 1, p. 195-217, 2016.

CIONI, G.; INGUAGGIATO, E.; SGANDURRA, G. Early intervention in neurodevelopmental disorders: underlying neural mechanisms. Developmental Medicine \& Child Neurology, v. 58, p. 61-66, 2016.

DE LANGE, A.-M. G. et al. The temporal dynamics of brain plasticity in aging. Cerebral Cortex, v. 28, n. 5, p. 1857-1865, 2018.

DEKKER, S. et al. Neuromyths in education: prevalence and predictors of misconceptions among teachers. Frontiers in Psychology, v. 3, p. 429, 2012.

DRAGANSKI, B. et al. Neuroplasticity: changes in grey matter induced by training. Nature, v. 427, n. 6.972, p. 311, 2004. 
DUMONTHEIL, I. Adolescent brain development. Current Opinion in Behavioral Sciences, v. 10, p. 39-44, 2016.

DUZEL, E.; VAN PRAAG, H.; SENDTNER, M. Can physical exercise in old age improve memory and hippocampal function? Brain, v. 139, n. 3, p. 662-673, 2016.

GEORGIEFF, M. K.; BRUNETTE, K. E.; TRAN, P. V. Early life nutrition and neural plasticity. Development and Psychopathology, v. 27, n. 2, p. 411-423, 2015.

GLEICHGERRCHT, E. et al. Educational neuromyths among teachers in Latin America. Mind, Brain, and Education, v. 9, n. 3, p. 170-178, 2015.

GOSWAMI, U. Neuroscience and education: from research to practice? Nature Reviews Neuroscience, v. 7, n. 5, p. 406, 2006.

GROSSI, M. G. R.; LOPES, A. M.; COUTO, P. A. A neurociência na formação de professores: um estudo da realidade brasileira. Revista da FAEEBA - Educação e Contemporaneidade, v. 23, n. 41, 2014.

HALE, J. et al. Critical issues in response-to-intervention, comprehensive evaluation, and specific learning disabilities identification and intervention: An expert white paper consensus. Learning Disability Quarterly, v. 33, n. 3, p. 223-236, 2010.

HALE, J. B. et al. Reconciling individual differences with collective needs: the juxtaposition of sociopolitical and neuroscience perspectives on remediation and compensation of student skill deficits. Trends in Neuroscience and Education, v. 5, n. 2, p. 41-51, 2016.

HERCULANO-HOUZEL, S. Do you know your brain? A survey on public neuroscience literacy at the closing of the decade of the brain. The Neuroscientist, v. 8, n. 2, p. 98-110, 2002.

HIDI, S. Revisiting the role of rewards in motivation and learning: implications of neuroscientific research. Educational Psychology Review, v. 28, n. 1, p. 61-93, 2016.

HOWARD-JONES, P. A. Neuroscience and education: myths and messages. Nature Reviews Neuroscience, v. 15, n. 12, p. 817, 2014.

KRAUSE, A. J. et al. The sleep-deprived human brain. Nature Reviews Neuroscience, v. 18, n. 7 , p. 404, 2017.

LINDENBERGER, U.; WENGER, E.; LÖVDÉN, M. Towards a stronger science of human plasticity. Nat. Rev. Neurosci, v. 18, p. 261-262, 2017.

LOPEZ, R. B. et al. A balance of activity in brain control and reward systems predicts selfregulatory outcomes. Social Cognitive and Affective Neuroscience, v. 12, n. 5, p. 832-838, 2017.

MARVIN, C. B.; SHOHAMY, D. Curiosity and reward: valence predicts choice and information prediction errors enhance learning. Journal of Experimental Psychology: General, v. 145, n. 3, p. 266, 2016. 
OLSON, R. K. Genes, environment, and reading disabilities. In: OLSON, R. K. Perspectives on learning disabilities. Abingdon: Routledge, 2018. p. 3-21.

PAPADATOU-PASTOU, M.; HALIOU, E.; VLACHOS, F. Brain knowledge and the prevalence of neuromyths among prospective teachers in Greece. Frontiers in Psychology, v. 8, p. 804, 2017.

RACINE, E. et al. Contemporary neuroscience in the media. Social Science \& Medicine, v. 71, n. 4, p. 725-733, 2010.

RAVER, C. C.; BLAIR, C. Neuroscientific insights: attention, working memory, and inhibitory control. The Future of Children, v. 26, n. 2, p. 95-118, 2016.

ROYAL SOCIETY. Brain Waves Module 2: Neuroscience: implications for education and lifelong learning. London: The Royal Society, 2011.

THOMAS, M. S. Essay review: limits on plasticity. Journal of Cognition and Development, v. 4, n. 1, p. 99-125, 2003.

UNIVERSIDADE FEDERAL DA GRANDE DOURADOS. Especialização em Educação Especial. 2019. Disponível em: https://portal.ufgd.edu.br/pos-graduacao/educacaoespecial/index. Acesso em: 15 jan. 2019.

VON STUMM, S.; PLOMIN, R. Monozygotic twin differences in school performance are stable and systematic. Developmental Science, v. 21, n. 6, p. e12694, 2018.

WILSON, R. et al. Educational attainment and cognitive decline in old age. Neurology, v. 72, n. 5, p. 460-465, 2009.

ZAHODNE, L. B.; STERN, Y.; MANLY, J. J. Differing effects of education on cognitive decline in diverse elders with low versus high educational attainment. Neuropsychology, v. 29, n. 4, p. 649, 2015.

\section{Como referenciar este artigo}

GAMA, Daniel Traina e FERRACIOLI, Marcela de Castro. Neurociência na educação especial: dos neuromitos às práticas pedagógicas baseadas em evidências. Doxa: Rev. Bras. Psico. e Educ., Araraquara, v. 21, n. 2, p. 285-296, jul./dez. 2019. e-ISSN: 2594-8385. DOI: https://doi.org/10.30715/doxa.v21i2.13101

Submetido em: 20/02/2019

Aprovado em: 20/07/2019

Publicado em: 01/08/2019 